\title{
FACTORES QUE INFLUYEN EN LA CRONOLOGÍA DE ERUPCIÓN DE LOS DIENTES PERMANENTES *
}

\author{
${ }^{1}$ Angélica Dabeiba Sanabria D., ${ }^{1}$ Diana Marcela Jiménez G., ${ }^{1}$ Tulia Inés Gutiérrez R. \\ ${ }^{2}$ Giovanna Pilonieta 0. \\ ${ }^{1}$ Estudiante de IX semestre, $F$ de Odontología, U. Santo Tomas, ${ }^{2}$ Odontóloga, U. Santo Tomás, \\ Especialista en Odontopediatria, U. Javeriana, Docente, U. Santo Tomas
}

Autor responsable de correspondencia: Diana Marcela Jiménez $G$.

Dirección de correo electrónico: nanaotito@yahoo.com

\begin{abstract}
RESUMEN
Es importante conocer los posibles factores generales y locales que influyen en la secuencia y en la cronología de erupción de la dentición permanente. Entre estos factores se han observado la edad, el género, el peso, la talla, la higiene oral, la caries dental, la pérdida prematura de los dientes deciduos y las concentraciones sistémicas de flúor, además de algunas condiciones patológicas poco frecuentes y de desarrollo. El objetivo de esta revisión es detallar los posibles eventos que influyen en la secuencia y en la cronología de erupción de la dentición permanente, pues es relevante conocer el tiempo y la secuencia de erupción como medio esencial para el diagnóstico y el tratamiento acertado del paciente. [Sanabria AD, Jiménez DM, Gutiérrez TI, Pilonieta G. Factores que influyen en la cronología de erupción de los dientes permanentes. Ustasalud Odontología 2006; 5: 132 - 136]
\end{abstract}

Palabras clave: Secuencia de erupción, Cronología de erupción.

\section{FACTORS THAT INFLUENCE THE CHRONOLOGY OF ERUPTION OF THE PERMANENT DENTITION}

\begin{abstract}
It is important to know the possible general and local factors that influence the sequence and the chronology of eruption of permanent teeth. Among these factors it had been observed the age, the gender, the weight, the height, the oral hygiene, the premature decay, the premature teeth lost and the fluoride concentrations; in addition, some pathological conditions concerned with the individual development. The objective of this paper is to detail the possible events that influence the sequence and the chronology of eruption of the permanent teeth because it is essential to know the time and the sequence of eruption for a correct diagnosis and treatment.
\end{abstract}

Key words: Sequence of eruption, Chronology of eruption.

Recibido para publicación: 3 de abril de 2006. Aceptado para publicación: 13 de diciembre de 2006.

${ }^{*}$ Grupo de Investigación CYDUSTA.

\section{INTRODUCCIÓN}

Erupción es el proceso responsable del movimiento dentario desde la ubicación del diente en la cripta, a través del proceso alveolar, hacia la cavidad bucal hasta encontrar su posición en la oclusión con el antagonista. Este es un proceso dinámico acompañado de la formación radicular completa, el establecimiento del periodonto y el mantenimiento de la oclusión funcional. Ocurre en un amplio rango de edad y puede ser influenciado por algunos factores como: edad, género, talla, peso, caries dental, pérdida prematura de los dientes, concentraciones sistémicas de flúor, higiene oral, factores locales como hiperplasia o fibromatosis gingival, tumores, quistes y fisuras palatinas y condiciones sistémicas como alteraciones endocrinas, hipotiroidismo o entidades sindrómicas como displasia cleidocraneal y síndrome de Down, entre otros. ${ }^{t-3}$
Moslemi (2004) encontró que la genética, el género, el nivel socioeconómico y nutricional, así como factores hormonales y geográficos, alteran los patrones de erupción. Es importante tener en cuenta que estos factores pues varían de una población a otra, e influyen en el tiempo de erupción de la dentición permanente. ${ }^{3}$

El objetivo de este artículo fue revisar los posibles factores generales y locales que influyen en la secuencia y cronología de la erupción de la dentición primaria y permanente, debido a la importancia de conocer el tiempo y la secuencia de erupción como medio esencial para el diagnóstico de posibles alteraciones y permitir el planteamiento de métodos preventivos y terapéuticos en odontopediatría y ortodoncia interceptiva que actúen como guías de erupción o solucionen las posibles secuelas. Otro elemento a destacar es su empleo en la odontología forense para la identificación de la edad cronológica aproximada en niños con registro de nacimiento desconocido. 


\section{Factores Generales}

\subsection{Edad}

Es útil tener presente la edad promedio para determinar si hay adelantos o retrasos notorios en el proceso eruptivo. La edad es importante pues los dientes permanentes erupcionan entre los 5 a los 13 años, excepto los terceros molares sirviendo como punto de referencia para un diagnóstico oportuno de anomalías. ${ }^{2-12}$

\subsection{Género}

La aparición de los dientes es más temprana en las niñas que en los niños. ${ }^{13.14}$ Lew encontró una diferencia significativa entre los dos géneros para la erupción de caninos y premolares. ${ }^{15}$ Por su parte, Kochhar y Richardson encontraron que las niñas, efectivamente, presenta erupción más temprana de todos los dientes con excepción del segundo molar. ${ }^{2}$

Según Diamanti y Townsed, en las niñas, el promedio de tiempo de erupción es 4.5 meses más temprano para el maxilar superior y 5.3 meses, para el inferior. ${ }^{16}$ Sin embargo, para ambos sexos, los dientes tienen la misma secuencia y el tiempo de erupción es más temprano para los dientes inferiores que para los superiores. 2,15

\subsection{Nutrición}

Es un factor vital en el crecimiento y el desarrollo de las estructuras orales y en la conservación de los tejidos orales. Durante los periodos de crecimiento celular rápido, las deficiencias nutricionales pueden tener un efecto irreversible en los tejidos orales en desarro1lo, por el contrario, si estas deficiencias se presentan después del desarrollo inicial de este órgano y sus tejidos circundantes, el efecto será reversible. Antes de la erupción dental, la nutrición puede influir en la maduración y composición química del esmalte, así como en la morfología y tamaño de los dientes. ${ }^{1,6,17}$

Los nutrientes para los cuales las insuficiencias o excesos se han relacionado directamente con trastornos orales son las proteínas, energía, vitaminas C, A y D, yodo y fluoruro. ${ }^{1,6}$

\subsection{Factores genéticos}

Es evidente la influencia genética en la formación de los huesos y de los dientes por ello se puede afirmar que hay malposiciones heredadas. Genéticamente, se hereda el tamaño y la forma de los dientes y de los huesos lo que ocasiona que se repitan algunos patrones morfológicos establecidos.

Algunas de las entidades relacionadas con alteraciones en la erupción dentaria son las siguientes:

\subsubsection{Síndrome Trisomía 21 (Síndrome de Down)}

El Síndrome Trisomía 21 es una de las anomalías congénitas en la que, frecuentemente, se presentan alteraciones en la erupción dentaria. Los primeros dientes temporales pueden erupcionar hacia los dos años de edad, completándose la dentición a los 40 5 años. La secuencia de erupción puede ser anormal y alguno de los dientes temporales podría permanecer hasta los 14 a 15 años de edad. El retardo en la erupción puede estar relacionado con una pobre vascularización del tejido conectivo periradicular 0 alteraciones en el crecimiento y desarrollo del complejo maxilomandibular..$^{18,19}$

Silva y Aguiar compararon la cronología de erupción en 113 niños con síndrome de Down comparado con 113 niños sin síndrome. Además, de lo que se ha encontrado en otros estudios, observaron que los incisivos laterales permanentes superiores e inferiores presentaban una erupción retardada en los niños con síndrome de Down. ${ }^{19}$

\subsubsection{Disostosis cleidocraneal}

La transmisión de la afección, conocida también como displasia osteodentinaria, displasia cleidocraneal, disostosis mutacional o síndrome de Marie-Sainton, se realiza a través del padre o de la madre a su hijo de género masculino o femenino siguiendo un autentico patrón mendeliano dominante. El síndrome también puede ocurrir en forma esporádica sin aparente influencia hereditaria y sin predilección racial. El diagnostico se basa en el hallazgo de la falta de las clavículas, pese a que pueden haber vestigios. ${ }^{6}$

El desarrollo de la dentición es retardada. No es raro observar una dentición temporal completa a los 15 años de edad a consecuencia de un retardo de la reabsorción radicular de los temporales y una demora en la erupción de los permanentes. Una de las características distintivas importantes es la presencia de dientes supernumerarios y la falta de cemento celular causando el retardo en la erupción; ${ }^{1}$ parece que se relaciona con seis tipos diferentes de mutaciones genéticas. ${ }^{20}$ Aunque se extraigan los dientes temporales y los supernumerarios, la erupción de la dentición permanente con frecuencia está demorada y es irregular. ${ }^{6}$

\subsubsection{Síndrome de Garner:}

Presenta la proliferación de sustancia similar al cemento y obliteración del espacio del ligamento periodontal ocasionando anquilosis

La erupción dental esta regulada por citoquinas, factor de crecimiento epidermal, factor de crecimiento $b$ transformante, interleuquina -1 y factor -1 estimulante de colonias. La falta de una apropiada respuesta inflamatoria, una inadecuada expresión de algunas citoquinas y el incremento de la densidad ósea que impide la reabsorción fisiológica han sido sugeridas como los factores causantes del retardo en la erupción dentaria en entidades sindrómicas. ${ }^{1}$ 


\subsection{Alteraciones sistémicas}

\subsubsection{Hipotiroidismo}

El hipotiroidismo congénito es el resultado de una ausencia o subdesarrollo de la glándula tiroides. El desarrollo de la dentición es lento incluyendo la erupción y exfoliación de los dientes temporales y la erupción de los permanentes. Los dientes son de tamaño normal con raíces más cortas pero están apiñados por la discrepancia dentoalveolar. El apiñamiento de los dientes, la maloclusión y la respiración bucal producen una gingivitis hiperplásica crónica. ${ }^{1,6}$

En los casos de hipotiroidismo juvenil no tratados, el retardo en la exfoliación de los dientes temporales y en la erupción de los dientes permanentes es característico. Un niño con edad cronología en los 12 años puede tener su dentición en un estado de desarrollo comparable con el de un niño de 9010 años de edad., 12

\subsubsection{Hipopituitarismo}

Causado por la deficiencia en la producción de la hormona de crecimiento, ocasionando así el retardo en el crecimiento de los huesos y los tejidos blandos del cuerpo. El retardo de la erupción es característico. En casos severos, en los dientes temporales no se presenta reabsorción radicular y se mantienen durante toda la vida. Los dientes permanentes subyacentes continúan desarrollándose pero no erupcionan. ${ }^{1,6}$

Kjellberg y colaboradores sugirieron que, en estos pacientes, la edad dental definida como madurez dental fue demorada en 1.2 años con respecto al grupo control. La erupción dental fue demorada un promedio de 1.3 años comparado con el grupo control. ${ }^{21}$

\subsubsection{Enanismo acondroplásico}

El crecimiento de las extremidades es limitado a causa de una falta de calcificación en el cartílago de los huesos largos. La cabeza es desproporcionadamente grande pero el tronco es de tamaño normal. Las fontanelas están abiertas en el momento del nacimiento. La parte superior de la cara esta subdesarrolla y el puente de la nariz esta deprimido. ${ }^{6}$

En algunos individuos resulta un crecimiento deficiente de la base del cráneo. El maxilar superior puede ser pequeño, con el consecuente apiñamiento de los dientes. El desarrollo de la dentición es levemente demorado. ${ }^{6}$

\section{Factores Sociales}

Se consideran como factores de riesgos sociales: el estrato socioeconómico, el estilo de vida y el nivel sociocultural. De los tres, se ha observado que el estrato socio-económico puede relacionarse con la cronología de la erupción.

\subsection{Estrato socioeconómico}

Se define como la asociación entre el nivel educativo, ingresos y ocupación de la persona.
Un individuo de estrato socioeconómico bajo tiene menos acceso a recursos que faciliten la salud, alimentación, casa y educación básica. ${ }^{9}$ La dificultad en el acceso a los servicios para consultas regulares, por no tener afiliación a la seguridad o pertenecer al régimen subsidiado y la falta de dinero imposibilita a este grupo de personas a obtener los servicios adecuados de salud oral, promoción y prevención, influyendo sobre el tipo de higiene de cada uno de los individuos; y su conocimiento sobre la importancia de la salud oral. ${ }^{22,23}$ Rowe y colaboradores reportaron un ligera demora en la erupción de los dientes permanentes en niños de estrato socioeconómico bajo. ${ }^{24}$

\section{Factores Locales}

\subsection{Obstrucción física}

La obstrucción física es la causa más común de alteraciones en la erupción. Estas obstrucciones pueden resultar de la presencia de supernumerarios, quistes, tumores odontogénicos y no odontogénicos, erupción ectópica, apiñamiento dentario, anquilosis del deciduo, no reabsorción radicular del temporal. ${ }^{1,17}$

Los dientes anquilosados están en un estado de retención estática, mientras que en las zonas adyacentes la erupción y el crecimiento son normales. ${ }^{20,25}$ Es común que se presente en la dentición temporal. ${ }^{26,27}$

Además de la anquilosis, la hiperplasia gingival resultante de varias causas (hormonales o hereditarias, deficiencia de vitamina c o el empleo de anticonvulsivos como fenitoina) también puede causar un denso tejido conectivo o colágeno acelular que alteraría la erupción dentaria. ${ }^{1}$

Las lesiones traumáticas pueden ocasionar erupción ectópica, alteraciones en la odontogénesis en forma de dilaceraciones o el desplazamiento del germen del permanente..$^{28}$

\subsection{Flúor sistémico}

El impacto de fluoruros en el tiempo de erupción de la dentición permanente se encuentra todavía en debate. Se ha sugerido que la incorporación de iones de flúor en el hueso alveolar produce un incremento en la resistencia a la reabsorción y conduce al retardo en la erupción del diente.

Campagna y colaboradores observaron que las niñas residentes en una zona con suministro de flúor sistémico presentaban un retardo en la erupción de acuerdo con su edad cronológica. ${ }^{29}$ En contraste, Virtanen y colaboradores encontraron un retardo mayor en la erupción de los dientes en los niños que residen en zonas fluoradas en comparación con las niñas. ${ }^{30}$

Leroy y colaboradores observaron un retraso en la erupción de la dentición permanente luego de que la dentición decidua fuera tratada con flúor como método preventivo ya que se disminuyó la pérdida de molares. ${ }^{31}$

134

Ustasalud Odontología 2006; 5:132 - 136

Sanabria A. y col. 


\subsection{Caries dental}

Se ha observado que la erupción de los dientes permanentes ocurre en edades más tempranas en áreas con alta prevalencia de caries lo que podría explicarse ya que la lesión cariosa puede contribuir a la aceleración de la pérdida prematura de los dientes deciduos afectados.

Leroy y colaboradores encontraron que la pérdida prematura debido a la caries de los molares primarios superiores aceleró la erupción de los premolares (10 a 19 meses), y en el maxilar inferior no hubo efectos significativos. $^{32}$

\subsection{Terapia pulpar}

En investigaciones ha sido revelado el índice significante en la aceleración en el proceso de erupción después de una pulpotomía; mientras Mc Donald \& Avery mencionaron que la pulpotomía puede causar retraso en la exfoliación y como una consecuencia, retraso en la erupción de los sucesores. ${ }^{1,6}$

Algunos estudios radiográficos han revelado que la caries dental, la necrosis pulpar y los tratamientos pulpares (pulpotomias) apresuraron el porcentaje de reabsorción radicular de los dientes deciduos, mientras que hubo influencia en el estado de formación radicular de los sucesores. La inflamación avanzada causa reabsorción de los dientes deciduos: la formación de abscesos seguido por reabsorción radicular contribuyen a la extracción temprana o exfoliación de los predecesores y aceleran la erupción de los premolares correspondientes. ${ }^{14,15}$

\subsection{Pérdida prematura de dientes deciduos}

La extracción de dientes primarios es un antecedente frecuente y un factor local influyente. La pérdida prematura de los dientes primarios, retarda la erupción de los dientes permanentes esto puede ser explicado por cambios anormales que pueden ocurrir en el tejido conectivo que rodea el permanente y la formación de una encía fibrosa y densa excepto para los premolares superiores, los cuales erupcionan más temprano., ${ }^{1,6,17}$

La erupción de los dientes permanentes inferiores se acelera si la extracción de los dientes primarios ocurre después de los 7.5 años de edad, pero se retarda si la extracción ocurre antes de esta edad. ${ }^{33}$

\subsection{Radiación}

Las alteraciones por radiación pueden estar relacionadas con anquilosis, alteración en la formación radicular, daño de las células del ligamento periodontal e insuficiencia del crecimiento mandibular causando disturbios en la erupción. ${ }^{34}$

\section{CONCLUSIONES}

El conocimiento sobre los factores que pueden alterar la secuencia de erupción de la dentición permanente, permite establecer parámetros que en un momento determinado ayudan a diagnosticar y plantear adecuados tratamientos.

Sobre el potencial biológico de crecimiento influyen múltiples factores, no sólo los relacionados con las circunstancias determinadas genéticamente sino con condiciones ambientales, sociales y locales.

La aparición de los dientes es más temprana en las niñas que en los niños, esta diferencia es evidente en relación con los caninos y premolares.

\section{BIBLIOGRAFÍA}

1. Suri L, Gagari E, Vastardis H. Delayed tooth eruption: Pathogenesis, diagnosis, and treatment. A literature review. Am JOrthod Dentofacial Orthop 2004; 126: 432 - 445 .

2. Kochhar R, Richardson A. The chronology and sequence of eruption of human permanent teeth in Northen Ireland. Int J Paediatr Dent 1998; 8: $243-252$

3. Wedl JS, Danias S, Schmelzle R, Friedrich RE. Eruption times of permanent teeth in children and young adolescents in Athens (Greece). Clin Oral Invest 2005; 9: 131 - 134.

4. Moslemi M. An epidemiological survey of the time and sequence of eruption of permanent teeth in 4 -15 year-olds in Tehran, Iran. Int J Paediatr Dent 2004; 14: 432 - 438.

5. Cárdenas D, Arias MI. Desarrollo de la Oclusión. En: Odontología pediátrica. 3ra ed. Medellín: Corporación para la Investigación Biológica; 2003. p. $275-294$.

6. McDonald RE, Avery D. Erupción de los dientes: factores locales, sistémicos y congénitos que influyen en el proceso. En: Odontología Pediátrica y del Adolescente. 6ta. Ed. Madrid: Mosby; 1990. p. $179-$ 207.

7. Van der Linden F. Ortodontia. Desenvolvimento da denticao. Quintessence Editora Ltda; 1986.

8. Leroy R, Bogaerts $\mathrm{K}$, Lesaffre E, Declerck D. The emergence of permanent teeth in Flemish children. Comm Dent Oral Epidemiol 2003; $31: 30$ - 39 .

9. Sarnat H, Kaffe I, Porat J, Amir E. Developmental stages of the third molar in Israeli children. Pediatr Dent 2003; 25: 373 - 377.

10. Ekstrand KR, Chistriansen J, Chistriancen ME. Time and duration of eruption of first and second permanent molar: a longitudinal investigation. Comm Dent Oral Epidemiol 2003; 31: 344 - 350.

11. Mugonzibwa EA, Kuijpers-Jagtman AM, Laine-Alava MT, Van't Hof MA. Emergence of permanent teeth in Tanzania children. Comm Dent Oral Epidemiol 2002; 30: 455 - 462.

12. Hitchcock N, Gilmour A, Gracey M, Kailis D. Australian longitudinal study of time and order of eruption of primary teeth. Community Dent Oral Epidemiol 1984; 12: 260 - 263. 
13. Hägg U. Taranger J. Dental development, dental age and tooth counts. Angle Orthod 1985; 55: $93-107$.

14. Demirjian A, Levesque GY. Sexual differences in dental development and prediction of emergence. J Dent Res 1980: 59: 1110 -1122 .

15. Lew KK. The prediction of eruption-sequence from panoramic radiographs. J Dent Child 1992: 346 - 349.

16. Diamanti J, Townsed GC. New standards for permanent tooth emergence in Australian children. Aust Dent J 2003; 48: $39-42$

17. Burdi AR, Moyers RE. Desarrollo de la dentición y la oclusión. En: Moyers RE. Manual de Ortodoncia. 4ta. Ed. Buenos Aires: Editorial Medica Panaméricana S.A.; 1992. p. $103-150$.

18. Ondarza A, Jara L, Muñoz P, Blanco R. Sequence of eruption of deciduous dentition in a Chilean sample with Down's syndrome. Arch Oral Biol 1997; 42: 401 - 406.

19. Silva KG, Aguiar SM. Erupção dental de crianças portadoras da sindrome de Down e crianças fenotipicamente normais: Estudo comparativo. Revista Odontológica de Araçatuba 2003; 24: 33 - 39.

20. Wise GE, Frazier-Bowers S, Souza RN. Cellular, molecular, and genetic determinants of tooth eruption. Crit Rev Oral Biol Med 2002; 13: $323-334$.

21. Kjeliberg $\mathrm{H}$, Beijing $\mathrm{M}$, Wickland $\mathrm{A}$. Craniofacial morphology, dental occlusion, tooth eruption, and dental maturity in boys of short stature with or without growth hormone deficiency. Eur J Oral Sci 2000; 108: 359 - 367

22. Norman O, Harris, García-Godoy F Odontología Preventiva Primaria. 2001. p. 102, 161-162, 235- 236.

23. Kals, Mc Donald, Stookey. Odontología Preventiva en Acción. 3era. Ed. 1990; cáp 7.

24. Rowe NH, Gran SM, Clark DC, Guire KE. The effect of age, sex, race, and economic status on dental caries experience of the permanent dentition. Committee to Review the Ten-State Nutrition Survey of 1968-1970. Pediatrics 1976; 57: 457 - 461 .

25. Delgado C, Moraes R. Anquilose Dento-Alveolar: Considerações sobre etiologia, diagnostico e posibilidades de tratamento. J Bras Odontopediatr Odontol Bebe 1999; 2: 167 - 174.

26. Osorio LM. Anquilosis de molares temporales: Revisión de la literatura y reporte de un caso. Ustasalud Odontología 2005; 4: 122 127.

27. Kocadereli I, Turgut M. Management of occlusal and developmental disturbances resulting from an ankylosed maxillary second primary molar: Case report. J Dent Child 2003; 70: 178 -181.

28. Andreasen Jo, Andreasen FM. Lesiones Dentarias Traumáticas. Madrid: Editorial Médica Panamericana; 1990.

29. Campagna L, Tsamtsouris A, Kavadia K. Fluoridated drinking water and maturation of permanent teeth at age 12. J Clin Pediatr Dent 1995; 19: 225 - 228.

30. Virtanen JI, Bloigu RS, Larmas MA. Timing of eruption of permanent teeth: standard Finnish patient documents. Community
Dent Oral Epidemiol 1994; 22: 286 - 288.

31. Leroy R, Bogaerts K, Lesaffre E, Declerck D. The effect of fluorides and caries in primary teeth on permanent tooth emergence. Comm Dent Oral Epidemiol 2003; 31: 463 - 470.

32. Leroy R, Bogaerts $\mathrm{K}$, Lesaffre E, Declerck D. Impact of caries experience in the deciduous molars on the emergence of the successors. Eur J Oral Sci 2003; 111: 106 - 110.

33. Kerr WJ. The effect of premature loss of deciduous canines and molars on eruption of permanent successors. Eur J Orthod 1980; 2: $123-128$.

34. Piloni MJ, Ubios AM. Impairment of molar tooth eruption caused by X-radiation. Acta Odontol Latinoam 1996; 9: $87-92$. 